\title{
Localised Solutions of Hartree Equations for Narrow-Band Crystals
}

Claudio Albanese ${ }^{\star}$

Theoretical Physics, ETH-Hönggerberg, CH-8093 Zürich, Switzerland

\begin{abstract}
We consider the Hartree equations for a system of an infinite number of electrons in a periodic potential consisting of a periodic array of wells. The filling fraction is assumed to be of one electron per well. We prove that if the wells are deep enough to admit a bound state and if they are separated by a distance large enough, then the Hartree equations have a solution in which all single particle wave functions decay exponentially.
\end{abstract}

\section{Introduction}

This note is dedicated to the study of the following eigenvalue problem

$$
\left\{\begin{array}{l}
-\Delta u+V u+W(u) u=E u \\
u \in W^{2,2}\left(\mathbb{R}^{3}\right),\|u\|_{2}=1, u(x)>0,
\end{array}\right.
$$

where

$$
V(x)=\sum_{i \in \mathbb{Z}^{3}} U_{a}(x+\underline{i} l)
$$

and $W(u)$ is the operator of multiplication times

$$
\sum_{\underline{i} \in \mathbb{Z}^{3} \backslash\{0\}} \int d y W(|y-x|) u(y+\underline{i} l)^{2} .
$$

Here $l$ and $a$ are positive parameters such that $l>2 a+\varepsilon$ for some fixed constant $\varepsilon>0, U_{a}(x)$ is the potential well

$$
U_{a}(x)=\left\{\begin{array}{rll}
-U & \text { if } & |x| \leqq a \\
0 & \text { if } & |x|>a
\end{array},\right.
$$

and $W(s)$ is a monotonously decreasing, nonzero function in $L^{\infty}\left(\mathbb{R}_{+}\right)$, such that

$$
0 \leqq W(s) \leqq C_{0} s^{-3-\eta} \quad \forall s \geqq 0
$$

for some constants $C_{0}, \eta>0$.

* Address after July, 1988: Department of Mathematics, University of California, Los Angeles, CA 90024, USA 
Equation (1.1) derives from the Hartree equations for an infinite number of electrons, one per well, moving in the background potential $V$ and interacting via the two body potential $W(|x-y|)$. In fact, if $u$ is a solution of (1.1), then $\left\{u_{i}(x)\right\}_{i \in \mathbb{Z} 3}$ with $u_{i}(x)=u(x-\underline{i} l)$, is a solution of the Hartree equations.

Our aim is to give a proof of the following result:

Theorem. Let us suppose that the potential well (1.4) is such that the operator $-\Delta+U_{a}(x)$ in $L^{2}\left(\mathbb{R}^{3}\right)$ has an isolated eigenvalue $\widetilde{E}$ at the bottom of its spectrum. Then, for all interaction potentials $W$ satisfying the conditions above, there is a constant $l^{*}$ such that if $l>l^{*}$, then Eq.(1.1) has a solution. This solution is exponentially decaying.

The existence of such a solution is related to the phenomenon of Mott's localization [1], which occurs when the bandwidth of the lowest band of $-\Delta+V$ is narrow enough. In this case, the repulsive effects of the interaction term get enhanced and, if the filling fraction is of one electron per atom, there is a solution of the Hartree equations in which each electron sits on a single well. In [2] we considered the full Schrödinger equation in the tight binding approximation for a system of scalar particles in a periodic background, and we found results in qualitative agreement with the picture given by the theorem above. We remark that the Hartree approximation is able to describe only the localization part of Mott's phenomenon, while all the very rich magnetic effects originating from the spin of the electron are completely ignored. See, however, [2] for an approximate treatment of the Hartree-Fock equations which permits us to incorporate the spin in the picture above.

The proof we give in the next sections is based on an approximation scheme which has been described in an abstract setting in [3]. We refer the reader to that paper for the proof of a theorem which will be stated in Sect. 2 and which represents the starting point of our proof.

\section{Strategy of the Proof}

Let $v_{0}$ be the ground state of the operator $-\Delta+U_{a}(x)$ on $L^{2}\left(\mathbb{R}^{3}\right)$, and let $\widetilde{E}$ be its eigenvalue. We shall use $v_{0}$ as an approximate solution of (1.1). According to the theorem in [3], if the linear eigenvalue problem

$$
\left\{\begin{array}{l}
(-\Delta+V) u_{0}+W\left(v_{0}\right) u_{0}=E_{0} u_{0} \\
u_{0} \in W^{2,2}\left(\mathbb{R}^{3}\right),\left\|u_{0}\right\|_{2}=1, u_{0}(x)>0
\end{array}\right.
$$

has a solution $\left(E_{0}, u_{0}\right)$ with $E_{0}$ simple and $\left\|u_{0}-v_{0}\right\|_{2}$ small enough, then (1.1) has a solution $u$ near $u_{0}$ in the $W^{2,2}$ norm. To give a more precise statement, let us set

$$
\begin{gathered}
\gamma=\operatorname{dist}\left(E_{0}, \sigma\left(-\Delta+V+W\left(v_{0}\right)\right) \backslash\left\{E_{0}\right\}\right), \\
\alpha_{0}=\gamma-\left\|\left(\frac{\partial}{\partial u} W\right)\left(u_{0}\right)[\cdot] u_{0}\right\|\left\|_{\mathscr{L}\left(L^{2}\right)}-4\right\| W\|\| u_{0}-v_{0} \|_{2}, \\
\alpha_{1}=1+7\|W\|+\left|E_{0}\right|, \\
\alpha_{2}=\left|E_{0}\right|+4\|W\|,
\end{gathered}
$$


where

$$
\|W\|=\sup _{x} \sum_{i \in \mathbb{Z}^{3}} W(|x+\underline{i} l|) .
$$

Thanks to the hypothesis expressed by (1.5), we have $\|W\|<\infty$. In [3] it is proven that if

$$
\alpha_{0}>0
$$

and

$$
\left\|u_{0}-v_{0}\right\| \leqq \frac{\alpha_{0}}{2 \alpha_{1}}\left[\exp \left(\frac{4 \alpha_{1}\|W\|}{\alpha_{0}}\left(1+\frac{1}{2} \alpha_{0}+\alpha_{2}\right)\right)-1\right]^{-1},
$$

then the problem (1.1) has a solution $u$ with

$$
\left\|u-u_{0}\right\| \leqq \frac{\alpha_{0}}{2 \alpha_{1}}
$$

In Sect. 3 we study the approximate equation (2.1) and give an upper bound for $\left\|u_{0}-v_{0}\right\|$ which tends to zero as $l \uparrow \infty$. We then verify that $\alpha_{0}$ and the right-hand side of (2.8) remain uniformly bounded from below by a positive constant as $l \uparrow \infty$. These two facts imply that as $l \uparrow \infty$, the two conditions (2.7) and (2.8) are eventually fulfilled. This proves the existence of a solution of the eigenvalue problem in (1.1).

From the proof of the theorem in [3], we can argue that the solution $u(x)$ satisfies also the positivity condition in (1.1). In fact, $u$ is constructed considering the following family of eigenvalue problems connecting (2.1) to (1.1):

$$
\left\{\begin{array}{l}
\left(-\Delta+V+W\left(v_{0}\right)\right) u_{\beta}+\beta\left(W\left(u_{\beta}\right)-W\left(v_{0}\right)\right) u_{\beta}=E_{\beta} u_{\beta} \\
u_{\beta} \in W^{2,2}\left(\mathbb{R}^{3}\right),\left\|u_{\beta}\right\|_{2}=1
\end{array}\right.
$$

where $\beta \in[0,1]$. In [3] we prove that if the conditions (2.7) and (2.8) are satisfied, then there is a smooth curve $\beta \mapsto u_{\beta} \in W^{2,2}\left(\mathbb{R}^{3}\right)$ of solutions of (2.10) such that $\left.u_{\beta}\right|_{\beta=0}=u_{0}$ and $\left.u \equiv u_{\beta}\right|_{\beta=1}$ fulfills (2.9). Moreover, $u_{\beta}$ is positive for all $\beta \in[0,1]$. In fact, from the proof in [3] it follows that $E$ is separated by a finite gap from the rest of the spectrum of the operator

$$
-\Delta+V+W\left(v_{0}\right)+\beta\left(W\left(u_{\beta}\right)-W\left(v_{0}\right)\right)
$$

for all $\beta \in[0,1]$. Hence, $u_{\beta}$ is the ground state of $(2.11)$ and it must be positive (see [4]). Thus, to prove the theorem in Sect. 1 it is enough to verify that the conditions (2.7) and (2.8) above are satisfied.

\section{Solution of the Approximate Equation (2.1)}

In this section we prove the existence of a solution $\left(E_{0}, u_{0}\right)$ of $(2.1)$. We shall use the so-called Dirichlet-Neumann bracketing trick, consisting in the use of the following operator inequalities:

$$
\left(-\Delta+V+W\left(v_{0}\right)\right)_{N} \leqq-\Delta+V+W\left(v_{0}\right) \leqq\left(-\Delta+V+W\left(v_{0}\right)\right)_{D},
$$


where $N$ (respectively $D$ ) denotes the insertion of Neumann (respectively Dirichlet) boundary conditions on the set $\Gamma$. $\Gamma$ is the union of periodically spaced planes with period $l$, such that the lattice $l \mathbb{Z}^{3}$ is the set of the centers of the cubes $Q(i),\left(i \in \mathbb{Z}^{3}\right)$, enclosed by $\Gamma$. Let us notice that the spectrum of the first and of the third operator of (3.1), is the union of the spectra $\left\{E_{n}^{N}(i)\right\}_{n \in \mathbb{N}}$ or $\left\{E_{n}^{D}(i)\right\}_{n \in \mathbb{N}}$ of the operators $(-\Delta+V$ $\left.+W\left(v_{0}\right)\right)_{N, D}$ restricted on the cubes $Q(i)$. To prove the existence of an eigenvalue at the bottom of the spectrum of $-\Delta+V+W\left(v_{0}\right)$, it is sufficient to prove that the following inequality holds:

$$
E_{0}^{D}(0)<\inf _{(n, i) \neq(0,0)} E_{n}^{N}(i) .
$$

In fact, in this case (3.1) implies that there is a simple and isolated eigenvalue $E_{0}$ of $-\Delta+V+W\left(v_{0}\right)$ in the interval $\left[E_{0}^{N}(\underline{0}), E_{0}^{D}(\underline{0})\right]$. In the following, we shall also prove some estimates which permit us to control $\left\|u_{0}-v_{0}\right\|_{2}$, the gap $\gamma$ and $\alpha_{0}$.

Let us introduce the following notations:

$$
\begin{aligned}
& \gamma^{N}(l)=\inf _{(n, i) \neq(0,0)} E_{n}^{N}(\underline{i})-E_{0}^{N}(\underline{0}), \\
& \gamma^{D}(l)=\inf _{(n, i) \neq(0,0)} E_{n}^{D}(\underline{i})-E_{0}^{D}(\underline{0}) .
\end{aligned}
$$

Lemma 3.1. (i) We have

$$
\gamma^{N} \equiv \inf _{l>2 a+\varepsilon} \gamma^{N}(l)>0 \quad \text { and } \quad \gamma^{D}=\inf _{l>2 a+\varepsilon} \gamma^{D}(l)>0
$$

Moreover, we have

$$
0<E_{0}^{D}(\underline{0})-\widetilde{E}<C_{1}(a, l, U),
$$

where $C_{1}(a, l, U)$ is a constant $\downarrow 0$ as $l \uparrow \infty$.

(ii) If $E_{0}^{D}(\underline{0})<0$, we have

$$
0<E_{0}^{D}(\underline{0})-E_{0}^{N}(\underline{0}) \leqq C_{3}(a, U, \varepsilon) \exp \left(\frac{1}{2} E_{0}^{D}(\underline{0}) l\right)
$$

for some constant $C_{3}(a, U, \varepsilon)$.

Proof. (i) This point is a consequence of the hypothesis that the operator $-\Delta+U_{a}(x)$ on $\mathbb{R}^{3}$ has an isolated eigenvalue $\tilde{E}$ at the bottom of its spectrum with gap $\tilde{\gamma}$. As $l \uparrow \infty$, the norm of the operator

$$
\sum_{i \neq 0} \int d y W(|y-x|) v_{0}(y+\underline{i} l)^{2}=\int d y \sum_{i \neq 0} W(|x-y-\underline{i} l|) v_{0}(y)^{2}
$$

converges to zero. Thus $\gamma^{N}(l)$ and $\gamma^{D}(l)$ tend to the minimum between $\tilde{\gamma}$ and the difference between $\widetilde{E}$ and the ground state energy of

$$
-\Delta+U_{a}(x)+\int d y W(|y-x|) v_{0}(y)^{2}
$$

on $\mathbb{R}^{3}$. Moreover, (3.6) follows from the fact that the difference between $E_{0}^{D}(0)$ and the ground state energy $\widetilde{E}_{0}^{D}(\underline{0})$ of $-\Delta+U_{a}(x)$ with Dirichlet conditions on $\partial Q(\underline{0})$ is smaller than the norm of the operator $(3.8)$, and that $\widetilde{E}_{0}^{D}(\underline{0})-\widetilde{E}$ decays exponentially fast as $l \uparrow \infty$. 
(ii) On the basis of a Green formula, we find

$$
\begin{aligned}
E_{0}^{D}(\underline{0})-E_{0}^{N}(\underline{0})= & \frac{1}{\left(u_{0 N}, u_{0 D}\right)}\left\{\int_{Q(\underline{0})} u_{0 N}\left[-\Delta+U_{a}(x)+W\left(v_{0}\right)\right] u_{0 D}\right. \\
& \left.+-\int_{Q(0)} u_{0 D}\left[-\Delta+U_{a}(x)+W\left(v_{0}\right)\right] u_{0 N}\right\} \\
= & \frac{-1}{\left(u_{O N}, u_{0 D}\right)} \oint_{\partial Q(Q)} u_{0 N} \frac{\partial u_{O D}}{\partial n},
\end{aligned}
$$

where $u_{O N}$ and $u_{O D}$ are the eigenfunctions corresponding to $E_{0}^{N}(\underline{0})$ and $E_{0}^{D}(\underline{0})$. The bound (3.7) will follow from an exponential decay estimate on $u_{0 N}$ and $\nabla u_{0 D}$. Let us consider first $u_{0 N}$. Let us rewrite the equation fulfilled by $u_{0 N}$ in integral form

$$
u_{O N}(x)=\int_{Q(0)} d y G_{N}(x, y)\left[U_{a}(y)+W\left(v_{0}\right)(y)\right] u_{0 N}(y),
$$

where $G_{N}(x, y)$ is the kernel of the operator $\left(-\Delta-E_{0}^{N}(\underline{0})\right)$ restricted to $Q(\underline{0})$ with Neumann boundary conditions on $\partial Q(\underline{0})$. The following explicit expression for $G_{N}(x, y)$ can be found with the method of images (see e.g. [5])

$$
G_{N}(x, y)=\sum_{j=0}^{\infty} G\left(x-y_{j}\right) \quad \forall x, y \in Q(\underline{0}),
$$

where $G$ is the kernel of the operator $\left(-\Delta-E_{0}^{N}(\underline{0})\right)$ on $\mathbb{R}^{3}$ and $\left\{y_{j}\right\} \subset \mathbb{R}^{3}$ is the set with $y_{0}=y$ which is invariant under all reflections with respect to the planes of $\Gamma$. We have

$$
G(x-y)=\frac{1}{4 \pi|x-y|} \exp \left(E_{0}^{N}(\underline{0})|x-y|\right) .
$$

From (3.4) we see that $G_{N}(x, y)>0$, so that from (3.3) we get the bound

$$
u_{0 N}(x) \leqq \int d y G_{N}(x, y) U_{a}(y) u_{0 N}(y) .
$$

If $|y| \leqq a$ and $x \in \partial Q(\underline{0})$, we have

$$
\begin{aligned}
G_{N}(x, y)= & \frac{1}{4 \pi} \sum_{j=0}^{\infty} \frac{1}{\left|x-y_{j}\right|} \exp \left(E_{0}^{N}(\underline{0})\left|x-y_{j}\right|\right) \\
\leqq & \frac{1}{4 \pi\left[\frac{1}{2} l-a\right]} \exp \left(E_{0}^{N}(\underline{0})\left(\frac{1}{2} l-a\right)\right)+\frac{1}{4 \pi} \sum_{i \neq 0} \frac{1}{|i| l-\frac{1}{2} l-a} \\
& \times \exp \left(E_{0}^{N}(\underline{0})\left(|\underline{i}| l-\frac{1}{2} l-a\right)\right) \leqq C_{2}(a, \varepsilon) l^{-1} \exp \left(\frac{1}{2} E_{0}^{N}(\underline{0}) l\right),
\end{aligned}
$$

where $C_{2}(a, \varepsilon)$ is a constant independent of $l$. Thus, if $x \in \partial Q(\underline{0})$, we find

$$
\begin{aligned}
u_{0 N}(x) & \leqq C_{2}(a, \varepsilon) l^{-1} \exp \left(\frac{1}{2} E_{0}^{N}(\underline{0}) l\right)\left(\int^{d y} U_{a}(y)^{2}\right)^{1 / 2} \\
& =C_{2}(a, \varepsilon)\left(\frac{4 \pi}{3} U^{2} a^{3}\right)^{1 / 2} l^{-1} \exp \left(\frac{1}{2} E_{0}^{N}(\underline{0}) l\right) .
\end{aligned}
$$

One can proceed analogously to bound $\nabla u_{0 D}$. In fact, also in this case the method of images is applicable to construct an explicit formula for the kernel $G_{D}(x, y)$ of the operator $\left(-\Delta-E_{0}^{D}\right)^{-1}$ restricted on $Q(\underline{0})$ with Dirichlet boundary conditions on $\partial Q(\underline{0})$. We have

$$
G_{D}(x, y)=\sum_{j=0}^{\infty} \varepsilon_{j} G\left(x-y_{j}\right),
$$


where $\varepsilon_{j}$ is the parity of the number of reflections with respect to the planes of $\Gamma$ needed to map $y_{0}$ into $y_{j}$. In this way we find a bound of the form (3.16) for $\underline{\nabla} u_{O D}$ with a slightly different constant.

To prove (3.7), it remains to show that $\left(u_{O N}, u_{0 D}\right)$ is bounded away from zero uniformly in $l$. This follows from the following general fact to which we appeal again below:

Sublemma 3.2. Let $H_{0}$ be a Schrödinger operator with a positive, nondegenerate ground state $u_{0} \geqq 0$ with energy $E_{0}$ separated by a gap $\gamma_{0}$ from the rest of the spectrum. Then, if $u(x)$ is a positive function such that $\left\|\left(H_{0}-E_{0}\right) u\right\| \leqq \varepsilon_{0}$, we have

$$
\left\|u-u_{0}\right\| \leqq \frac{\sqrt{2}}{\gamma} \varepsilon_{0} .
$$

Proof of the Sublemma. If $H_{0}=\int_{E_{0}}^{\infty} \lambda d P_{\lambda}$ is the spectral decomposition of $H_{0}$, we have

$$
\begin{aligned}
& \varepsilon_{0}^{2} \geqq\left\|\left(H_{0}-E_{0}\right) u\right\|^{2}=\left\|\int_{E_{0}+\gamma}^{\infty}\left(\lambda-E_{0}\right) d P_{\lambda} u\right\|^{2}=\int_{E_{0}+\gamma}\left|\lambda-E_{0}\right|^{2}\left\|d P_{\lambda} u\right\|^{2} \\
& \geqq \gamma^{2}\left\|u-\left(u, u_{0}\right) u_{0}\right\|^{2} \geqq \frac{1}{2} \gamma^{2}\left\|u-u_{0}\right\|^{2} \text {. Q.E.D. }
\end{aligned}
$$

From this sublemma we can argue that $\left(u_{0 N}, u_{O D}\right)$ is exponentially close to one. In fact, if $H_{0}$ is the operator $-\Delta+U_{a}(x)+W\left(v_{0}\right)$ restricted to $Q(\underline{0})$ with Neumann boundary conditions on $\partial Q(\underline{0})$, we have

$$
\left\|\left(H_{0}-E_{0}^{N}(\underline{0})\right) u_{0 D}\right\|=E_{0}^{D}(\underline{0})-E_{0}^{N}(\underline{0}),
$$

and the right-hand side is exponentially small thanks to the first part of the proof. Q.E.D.

This lemma has the following consequences:

Proposition. If $l$ is large enough, then Eq. (2.1) has a solution $\left(E_{0}, u_{0}\right)$ such that

$$
\gamma \geqq \gamma^{N}-C_{3}(a, U, \varepsilon) \exp \left(\frac{1}{2} E_{0}^{D}(\underline{0}) l\right),
$$

and there is a constant $C_{4}(a, U, \varepsilon)$ such that

$$
\left\|u_{0}-v_{0}\right\|_{2} \leqq C_{4}(a, U, \varepsilon) C_{1}(a, U, l) \text {. }
$$

Proof. To prove (3.20) one can use again Sublemma 3.2. In fact we have

$$
\begin{aligned}
\|( & \left.-\Delta+V+W\left(v_{0}\right)-E_{0}\right) v_{0} \|^{2} \leqq\left|\tilde{E}-E_{0}\right|^{2}+\sum_{i \neq 0} \int d x U_{a}(x+\underline{i} l)^{2} v_{0}(x)^{2} \\
& +\sup _{x \in \frac{1}{2} Q(Q)}\left[\sum_{i \neq 0} \int_{\frac{1}{2} Q(Q)} d y W(|y-x-\underline{i} l|) v_{0}(y)^{2}\right]^{2}+2\|W\| \int_{\mathbb{R}^{2} \backslash \frac{1}{2} Q(Q)} d x v_{0}(x)^{2} \\
\leqq & 2 C_{1}(a, U, l)^{2}+C_{5}(a, U) e^{\tilde{E} l}
\end{aligned}
$$

for some constant $C_{5}(a, U)$. Hence we find

$$
\left\|u_{0}-v_{0}\right\| \leqq\left(\gamma^{N}-C_{3}(a, U, \varepsilon) e^{\frac{1}{2} E_{0}^{D}(0) l}\right)^{-1}\left(4 C_{1}(a, U, l)^{2}+2 C_{5}(a, U) e^{\tilde{E} l}\right),
$$

whence (3.20) follows. Q.E.D. 
To complete the proof of the theorem in Sect. 1, what remains to do is to verify that (2.7) holds if $l$ is large enough. If $f \in L^{2}\left(\mathbb{R}^{3}\right)$ is such that $\|f\|_{2}=1$, we have

$$
\begin{aligned}
\left\|\left(\frac{\partial}{\partial u} W\right)\left(u_{0}\right)[\cdot] u_{0}\right\|_{\mathscr{L}\left(L^{2}\right)} & \leqq 2\left|\sum_{i \neq 0} \int d x d y W(|y-x|) u_{0}(y+i l) f(y+\underline{i} l) u_{0}(x) f(x)\right| \\
& =2\left|\int d x d y\left[\sum_{i \neq 0} W(|y-x-\underline{i} l|)\right] u_{0}(y) f(y) u_{0}(x) f(y)\right| \\
& \leqq 2\left|\int_{B} \ldots\right|+2\left|\int_{\mathbb{R}^{\sigma} \backslash B} \ldots\right|
\end{aligned}
$$

where $B=\left(\frac{1}{2} Q(\underline{0})\right) \times\left(\frac{1}{2} Q(\underline{0})\right)$. We have

$$
\left|\int_{B} \cdots\right| \leqq \sup _{x \in Q(0)} \sum_{i \neq 0} W(|x-\underline{i} l|) .
$$

Also

$$
\begin{aligned}
\left|\int_{\mathbb{R}^{6} \backslash B} \cdots\right| \leqq & \|W\|\left|\int_{\mathbb{R}^{\sigma} \backslash B} f(x) f(y) u_{0}(x) u_{0}(y)\right| \\
= & \|W\| \mid \int_{\mathbb{R}^{6 \backslash B}} f(x) f(y)\left[v_{0}(x) v_{0}(y)+\left(u_{0}(x)-v_{0}(x)\right) u_{0}(y)\right. \\
& \left.+\left(u_{0}(x)-v_{0}(x)\right) v_{0}(y)\right] \mid \\
\leqq & \|W\|\left[\int_{\mathbb{R}^{3} \backslash \frac{1}{2} Q(0)} v_{0}(x)^{2}+2\left\|u_{0}-v_{0}\right\|_{2}\right] .
\end{aligned}
$$

Thus we have

$$
\begin{aligned}
\alpha_{0}= & =\gamma-\sup _{x \in Q(0)} \sum_{i \neq 0} W(|x-\underline{i} l|)-\|W\| \int_{\mathbb{R}^{3} \backslash \frac{1}{2} Q(0)} v_{0}(x)^{2} \\
& -2\|W\|\left\|u_{0}-v_{0}\right\|_{2}-4\|W\|\left\|u_{0}-v_{0}\right\|_{2} .
\end{aligned}
$$

Thanks to (1.5), the exponential decay of $v_{0}(x)$ and (3.20), we find that $\alpha_{0}>0$ for $l$ large enough. The proof of the theorem is completed.

Acknowledgement. I would like to thank W. Hunziker for having pointed out an error in a preceeding version of this paper.

\section{References}

1. Mott, N.: Metal-insulator transitions, London: Taylor \& Francis 1974

2. Albanese, C.: ETH preprint 1988

3. Albanese, C.: A continuation method for non-linear eigenvalue problems. J. Funct. Anal. (to appear)

4. Reed, M., Simon, B.: Methods of modern mathematical physics. New York: Academic Press 1979

5. Glimm, J., Jaffe, A.: Quantum physics. Berlin, Heidelberg, New York: Springer 1981

Communicated by C. H. Taubes

Received September 21, 1987; in revised form June 8, 1988 
